\title{
Cerebellar attraction
}

The inferior olivary nucleus is a brainstem structure that projects to the Purkinje cells of the cerebellum. This olivocerebellar projection is known to be important for motor learning and the timing of movements. In a new study published in Developmental Biology, Zhu and colleagues turn their attention to its development - more specifically, how olivary axons are guided to the cerebellum. Zhu et al. present compelling evidence that the cerebellum itself produces long-range, diffusible guidance cues.

The olivary neurons originate in rhombomeres 7 and 8 of the hindbrain, and their axons grow across the ventral midline (floor plate) and project to the cerebellum on the contralateral side. It is thought that the growing axons are attracted to the floor plate by guidance molecules such as netrin-1, and their final topographic organization seems to be determined by local signals within the

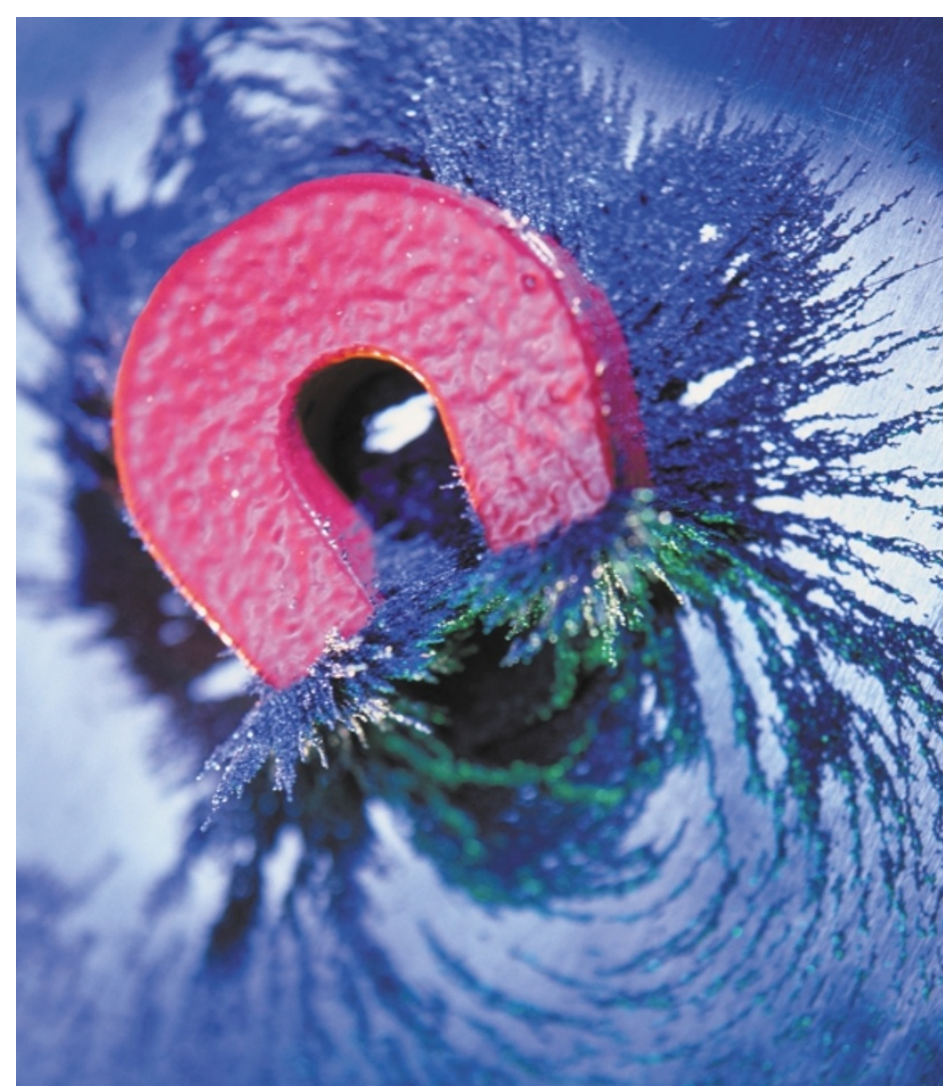

cerebellum that act over a short cues that guide the axons once they have crossed the midline have remained largely obscure.

Zhu et al. examined the effects of cerebellar tissue on olivary axon growth and guidance by transplanting a cerebellar plate alongside the hindbrain at the same rostrocaudal level as the inferior olivary nucleus. They found that neurons in the caudolateral part of the nucleus, which normally project to the rostral cerebellum, projected to the transplant and could make the correct topographic connections, even when the rostrocaudal polarity of the grafted tissue was reversed. However, the rostromedial olivary neurons, which normally project to the caudal cerebellum, could only make the right connections with the ectopic cerebellum if its polarity was reversed, so that their target was rostral to their cell bodies. This led the authors to suggest that range. However, the origins of the inhibitory signals in the caudal hindbrain might prevent olivary axons from projecting caudally. This idea was supported by experiments in which a cerebellum was grafted adjacent to the cervical spinal cord. In this case, none of the rostromedial neurons, and only a few of the caudolateral neurons, projected to the ectopic cerebellum.

So, the cerebellum clearly releases chemoattractants for olivary axons, but can these molecules diffuse over sufficiently long distances to be candidates for the endogenous cues? To find out, the authors sandwiched a slice of spinal cord tissue, which is normally non-permissive for olivary axon growth, between a caudal hindbrain explant and an explant of cerebellar tissue. They found that the olivary axons could cross the spinal cord 'bridge' to reach the cerebellum, indicating that the cerebellar chemoattractants act over a long range.

Like the axons of many other neuronal subtypes, the olivary axons change their sensitivity to guidance cues as they cross the midline. Zhu et al. found that exposure to floor plate signals made the axons more responsive to cerebellar guidance cues, possibly through upregulation of receptors for cerebellum-derived chemoattractants.

These findings show that the cerebellum not only produces locally acting signals that ensure that the olivary projections make the right topographic connections, but it also secretes long-range cues that guide olivary axons to the cerebellar primordium. The next step will be to identify these signals - a quest that will undoubtedly keep researchers occupied for some time to come.

Heather Wood

(1) References and links ORIGINAL RESEARCH PAPER Zhu, $Y$. et al.

Signals from the cerebellum guide the pathfinding of inferior olivary axons. Dev. Biol. 257, 233-248 (2003) FURTHER READING De Zeeuw, C. I. et al. Microcircuitry and function of the inferior olive. Trends Neurosci. 21, 391-400 (1998) | Wang, V. Y. \& Zoghbi, H. Y. Genetic regulation of cerebellar development. Nature Rev. Neurosci. 2, 484-491 (2003)
Two wrongs make a right

That mainstay of scientific advancement, the serendipitous discovery, has revealed a promising new treatment for ischaemic stroke. The chance combination of caffeine and alcohol in the lab of James Grotta at the Houston Medical School, University of Texas, prevents up to $80 \%$ of the brain damage caused by stroke in rats.

Grotta explained that "One of my laboratory workers, Roger Strong, was musing about the fact that moderate or mild use of alcohol can be effective in reducing the effect of stroke. So we started fooling around with combinations of it with other things ... and we stumbled on this" (HealthScoutNews, Switzerland, 10 April 2003).

Dubbed 'caffeinol', the

new combination has been used to treat 23 patients, 13 of which showed significant neurological improvement. In this small trial, no side effects were associated with its use. Importantly, caffeinol can be administered in combination with clot-busting drugs.

"We are at the phase where we think that the combination is more effective than any other drugs you look at in the laboratory, particular for strokes that affect the cortex - the grey matter of the brain that controls language and other abilities," said Grotta. "Often, it is the part of the brain that hangs in the balance after a stroke" (Health24 News, South Africa, 11 April 2003). Caffeinol is administered intravenously and - as with other stroke treatments the earlier treatment commences, the better. So, to those stroke sufferers that might be tempted to selfmedicate with a wee dram and a short black, doctors issue this warning: "No one who is having a stroke should take either caffeine or alcohol and ... people should get to the hospital immediately" (Sydney Morning Herald, Australia, 14 April 2003).

Suzanne Farley 